\title{
Cost-Effectiveness Analysis of Single-Use EEG Cup Electrodes Compared with Reusable EEG Cup Electrodes
}

\author{
Anne Sohrt ${ }^{1} \cdot$ Anders Mærkedahl $^{1}$ (1) $\cdot$ William V. Padula ${ }^{2}$
}

Published online: 27 August 2018

(c) The Author(s) 2018, corrected publication September 2018

\begin{abstract}
Background Hospital-acquired infections are one of the most frequent adverse events among patients receiving healthcare. Reusable electroencephalography cup electrodes (EEG-CE) pose a risk of infection due to cross-contamination, which can be eliminated by replacing reusable EEG-CE with single-use EEG-CE.

Objective The objective was to investigate the cost effectiveness of using single-use EEG-CE instead of reusable EEG-CE when the risk of sepsis is considered.

Methods A cost-effectiveness analysis was conducted, using a decision analytic model to assess the potential effects and costs of using single-use EEG-CE as an alternative to reusable EEG-CE. The cost data regarding the reusable EEG-CE were assessed through interviews with four hospitals. To identify the risk of sepsis, a retrospective observational cohort study was conducted using data from Truven Health Analytics, with a total of 73,834 patients analyzed in the USA during 1 February 2014 through 15 December 2014. A probabilistic sensitivity analysis (PSA) was conducted to test the impact of the variables simultaneously and the robustness of the result.

Results The incidence of sepsis was 33 cases per 100,000 EEG procedures. The acquisition and reprocessing costs of reusable EEG-CE were \$US3.25 and \$US5.57, respectively. The base-case analysis showed that single-use EEG-CE are cost effective compared with reusable EEG-CE, although the decision was not robust, with the PSA showing that $40 \%$ of the incremental cost-effectiveness ratios indicated that single-use EEG-CE were more effective but more expensive.

Conclusion This study indicates single-use EEG-CE are associated with cost savings and a possible reduction in the risk of cross-contamination.
\end{abstract}

\section{Key Points for Decision Makers}

Single-use electroencephalography (EEG) cup electrodes are likely cost effective compared with reusable EEG cup electrodes.

Use of single-use EEG cup electrodes may be associated with overall hospital savings.

Electronic supplementary material The online version of this article (https://doi.org/10.1007/s41669-018-0090-3) contains supplementary material, which is available to authorized users.

Anders Mærkedahl

andm@ambu.com

Ambu A/S, Baltorpbakken 13, 2750 Ballerup, Denmark

2 Department of Health Policy and Management, Johns Hopkins Bloomberg School of Public Health, $624 \mathrm{~N}$ Broadway Ave \#456, Baltimore, MD 21205, USA

\section{Background}

Hospital-acquired infections (HAI) are among the most frequent adverse events in patients receiving healthcare and result in a considerable economic burden for healthcare systems [1,2]. Given the economic burden and the discomfort of patients, such infections are undesirable. Reusable medical devices pose a risk of infection due to cross-contamination, where an infectious agent is carried from one person to another. This can occur directly from patient to patient or indirectly through a contaminated person or object [3]. Replacing reusable devices with single-use devices is one method of bypassing the risk of infection due to crosscontamination from medical devices.

Electroencephalography cup electrodes (EEG-CE) are available in both reusable and single-use versions. The single-use version makes it possible to eliminate the risk of cross-contamination [4]. EEG is a common diagnostic tool in the clinic, utilized on suspicion of epilepsy, 
unexplained unconsciousness, meningitis, and some types of dementia [5]. Before applying EEG-CE to the scalp, the skin is cleaned and an abrasive paste is used to remove the epidermal layer of the skin to lower the impedance level $[6,7]$. Because of this abrasion of the skin, EEG-CE are categorized as semi-critical devices. A semi-critical device is defined as one that contacts mucous membranes or nonintact skin [8-10]. This requires EEG-CE to be high-level disinfected (HLD) or sterilized [9, 10]. Reusable EEG-CE poses a risk of cross-contamination from inadequate reprocessing. If contaminated electrodes are used on a patient, there is a risk of infection. A 2018 study investigating pathogen growth and risk of contamination on cleaned readyto-use EEG-CE at four US hospital centers revealed 25\% of EEG-CE had positive bacterial culture. Of the bacteria found, $88 \%$ had the potential for or a risk of infection [11]. Many different infections can lead to sepsis, and $11 \%$ of all sepsis cases start with a skin infection. Sepsis is a severe syndrome that can lead to tissue damage, organ failure, and death and is very expensive to treat $[12,13]$.

Limited literature is available concerning both the risk of infection when using reusable EEG-CE and the costs and benefits of reusable EEG-CE compared with single-use EEG-CE. The objective of this study is to investigate the cost effectiveness of using single-use EEG-CE instead of reusable EEG-CE when considering the risk of sepsis.

\section{Methods}

A decision analytic model was constructed to assess the potential effects and costs of single-use EEG-CE compared with reusable EEG-CE (Fig. 1). The study was based on data from the USA. All costs are presented in \$US, year 2016 values, and are based on the retail acquisition price of the EEG-CE. Costs that were not obtained in 2016 values were adjusted using the consumer price index from Trading Economics [14]. Only direct costs were included, and elements with no relation to the EEG procedures and potential subsequent infections were excluded. Only costs that differed between the two types of electrodes were included.

This study was from a healthcare sector perspective, where the extra costs associated with sepsis acquired due to an EEG procedure were included and the time horizon was 1 year. The decision model was constructed using the dedicated software TreeAgePro 2015 (Fig. 1).

\subsection{Model Input}

To identify the risk of sepsis, a retrospective observational cohort study was designed using data from Truven Health Analytics MarketScan ${ }^{\circledR}$ Commercial Claims and Encounters and Medicare Supplemental Databases. Truven MarketScan ${ }^{\circledR}$ includes $>43.6$ million de-identified patientlevel healthcare claims data from managed care organizations, hospitals, electronic medical records providers, Medicare, and Medicaid [15]. The same patient was investigated during two outpatient visits: one visit with one or more outpatient medical claim for EEG, and a second visit with one or more medical claim not related to EEG at least 30 days before the outpatient medical claim for EEG. Patient claims from 1 February 2014 through 15 December 2014 were followed. The date of the earliest outpatient medical claim for EEG was considered the EEG index date, and the date of the latest outpatient visit not related to EEG before the EEG index date was considered the non-EEG index date. A sepsis case was registered if the patient had acquired sepsis 14 days after the EEG index date. Patients were excluded if they had outpatient medical claims for infection during the 30-day period before the EEG or the non-EEG index date. Patients

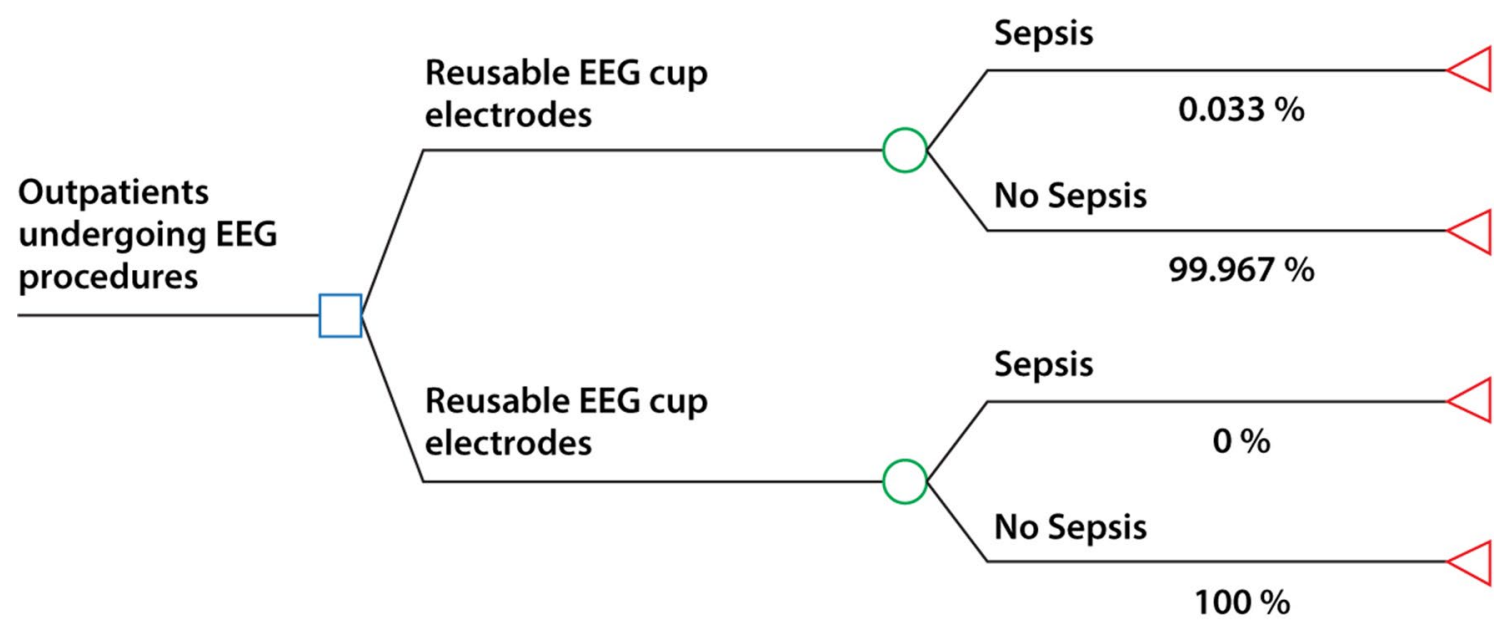

Fig. 1 Decision analytic model for cost effectiveness of single-use vs. reusable electroencephalography (EEG) cup electrodes 
were also excluded if they had inpatient or outpatient medical claims for surgery on the EEG or non-EEG index date or during the 14-day period after the EEG or non-EEG index date. Sepsis was defined if a patient had a diagnosis of (1) systemic infection (primary position; International Statistical Classification of Diseases and Related Health Problems, ninth edition [ICD-9] 0.38.xx) and sepsis (secondary position; ICD-9 995.91) or (2) systemic infection (primary position; ICD-9 0.38.xx), severe sepsis (secondary position; ICD-9 995.92). Only the costs of sepsis, and not those of minor infections, were included in the model, as sepsis has the greatest economic impact.

Effectiveness was measured in reduced infection rates among patients who had undergone an EEG procedure. The probabilities of the chance nodes reflect the uncertainties associated with the effect measure. It was assumed that the risk of infection was $0 \%$ when using single-use EEG-CE.

The retail acquisition costs and reprocessing costs for reusable EEG-CE were obtained from four US hospital sites, selected because of their diversity in location (east, midwest [north], south, and west), diversity in size (from 500 beds to $>1400$ beds), presence of an epilepsy monitoring unit, and use of reusable EEG electrodes as part of usual care assessment. Data were collected from 6 March to 24 April 2017. Department staff were interviewed about the time spent on reprocessing and the costs related to the acquisition and reprocessing of the electrodes. All four hospitals were utilizing HLD as the reprocessing method. The cost of reprocessing was based on assumptions about the amounts of detergent and disinfectant used (two disinfection wipes, $2 \mathrm{ml}$ detergent, and $379 \mathrm{ml}$ bleach per cleaning), and the data were supplemented by an internet search using the Google search engine to identify the acquisition costs of detergents and disinfectants. The retail acquisition cost of a single-use EEG-CE (Ambu ${ }^{\circledR}$ Neuroline Cup) was estimated at \$US18.50 per procedure, based on average selling price, provided by Ambu A/S [16].

The cost per sepsis case was determined from the literature, specifically, a 2016 systematic review assessing hospital-related costs of sepsis [12]. Based on 37 studies, including $>9$ million patients, the mean hospital-wide cost based on sepsis per patient was \$US33,718.

\subsection{Sensitivity Analyses}

Several one-way sensitivity analyses were conducted to test the impact on the incremental cost-effectiveness ratio (ICER) of varying the risk of sepsis and the costs of reusable EEG-CE and single-use EEG-CE, respectively. A twoway sensitivity analysis was conducted to test the impact of price levels for each of the technologies. Here, we used a total cost for use of reusable EEG-CE, so the acquisition and reprocessing cost was added. A probabilistic sensitivity analysis (PSA) was conducted to test the impact of the variables simultaneously and the robustness of the result. A second-order Monte Carlo simulation with 10,000 simulations was used. Gamma distributions were applied to the cost variables because of their typical right-skewed nature and because this parameter can only take a positive value. As the probabilities can only be between one and zero, a Beta distribution was selected.

\section{Results}

A total of 73,834 patients were included in the retrospective observational cohort study estimating the risk of sepsis. Demographic characteristics of the study cohort are shown in Table 1. No patients had acquired sepsis 14 days after the non-EEG index date, whereas 24 of 73,834 patients had acquired sepsis 14 days after the EEG index date (33 sepsis cases per 100,000 EEG procedures). The difference in risk of sepsis after the EEG index date and after the non-EEG index date was significant $(p<0.001)$.

The average time spent reprocessing per EEG procedure was $11.66 \mathrm{~min}$ (range 10-13.5), and the average hourly wage for the person conducting the reprocessing was \$US25 (range 19.9-31.5), giving an average wage cost per reprocessing of \$US4.86. The average cost for materials used for reprocessing was $\$$ US0.71 (range 0.40-1.04), giving a total cost per reprocessing of \$US5.57. The average cost of use of the reusable EEG-CE was based on an average of 25 electrodes used per procedure and the estimated number of times the reusable EEG-CE can be reused. The estimated number of reuses was 82, based on the annual number of EEG procedures and the annual number of purchased reusable EEG$\mathrm{CE}$ at the four hospitals, resulting in an average cost of use for reusable EEG-CE of \$US3.25 (range 0.25-9.79). See Table 2 for all model inputs.

The base-case analysis showed an average cost of reusable EEG-CE of \$US19.95 and an incidence of sepsis of 33 cases per 100,000 EEG procedures. The average cost of single-use EEG-CE was \$US18.50, and the risk of

Table 1 Demographic characteristics of the study cohort $(n=73,834)$

\begin{tabular}{ll}
\hline Characteristic & \\
\hline Age, years & $41.0 \pm 24.9$ \\
Age categories, years & \\
$\quad<65$ & $59,982(81.2)$ \\
$\geq 65$ & $13,852(18.8)$ \\
Sex & \\
Male & $31,411(42.5)$ \\
Female & $42,423(57.5)$
\end{tabular}

Data are presented as mean \pm standard deviation or $\mathrm{n}(\%)$ 
Table 2 All model inputs: parameter values and their respective standard errors, distributions and sources

\begin{tabular}{llll}
\hline Parameter & Base-case value (SE) & Distribution & Source \\
\hline Effects & & & Truven Health Analytics \\
$\quad$ Sepsis risk with reusable EEG-CE & 33 sepsis cases per 100,000 EEGs (17) & Beta & NA \\
Sepsis risk with single-use EEG-CE & 0 sepsis cases per 100,000 EEGs (17) & Beta & Interviews \\
Cost (\$US, year 2016 values) & & Gamma & Interviews/Google search \\
Acquisition of reusable EEG-CE & $3.25(2.98)$ & Gamma & Producer (Ambu A/S) \\
Reprocessing of reusable EEG-CE & $5.57(0.94)$ & Gamma & Gamma \\
Acquisition of single-use EEG-CE & $18.50(3.70)$ & $33,718(5785)$ & (11) \\
Cost per case of sepsis & &
\end{tabular}

$E E G$ electroencephalography, $E E G-C E$ electroencephalography cup electrodes, $N A$ not applicable, $S E$ standard error

sepsis was $0 \%$. Thus, the base-case results indicated that the single-use EEG-CE was less costly and more effective than the reusable EEG-CE with regard to sepsis. Table 3 presents the base-case results and an overview of the different one-way sensitivity analyses performed.
Figures 2 and 3 show the results of the two-way sensitivity analyses, illustrating which of the two technologies are most advantageous when adjusting for cost of sepsis and sepsis rates for single-use and reusable EEG-CE, respectively. The PSA indicated that single-use EEG-CE were more effective in avoiding sepsis and less costly, as $62 \%$
Table 3 Base-case result and one-way sensitivity analyses

\begin{tabular}{|c|c|c|c|}
\hline Scenario & $\Delta$ cost $(\$ \mathrm{US})$ & $\Delta$ effect $^{\mathrm{a}}$ & ICER $^{\mathrm{b}}$ \\
\hline Base-case & -1.45 & 33 & -4394 \\
\hline \multicolumn{4}{|l|}{ One-way sensitivity analyses } \\
\hline \multicolumn{4}{|l|}{ Reusable EEG-CE } \\
\hline When number of reuses is 200 (acquisition \$US1.39) & 0.41 & 33 & 1242 \\
\hline When number of reuses is 150 (acquisition $\$$ US1.85) & 0.05 & 33 & 151 \\
\hline When number of reuses is 100 (acquisition \$US2.78) & -0.98 & 33 & -2970 \\
\hline When number of reuses is 50 (acquisition \$US5.55) & -3.75 & 33 & $-11,364$ \\
\hline When number of reuses is 30 (acquisition \$US9.25) & -7.45 & 33 & $-22,576$ \\
\hline 27 cases of sepsis per 100,000 EEG procedures & 0.58 & 27 & 2148 \\
\hline 30 cases of sepsis per 100,000 EEG procedures & -0.44 & 30 & -1467 \\
\hline 36 cases of sepsis per 100,000 EEG procedures & -2.46 & 36 & -6833 \\
\hline 39 cases of sepsis per 100,000 EEG procedures & -3.47 & 39 & -8897 \\
\hline When the cost of sepsis is $\$ \mathrm{US} 28,000$ & 0.44 & 33 & 1333 \\
\hline When the cost of sepsis is $\$ \mathrm{US} 31,000$ & -0.55 & 33 & -1667 \\
\hline When the cost of sepsis is $\$ \mathrm{US} 37,000$ & -2.53 & 33 & -7667 \\
\hline When the cost of sepsis is $\$ \mathrm{US} 40,000$ & -3.52 & 33 & $-10,667$ \\
\hline \multicolumn{4}{|l|}{ Single-use EEG-CE } \\
\hline When costs of single-use EEG-CE are \$US14.80 & -5.15 & 33 & $-15,606$ \\
\hline When costs of single-use EEG-CE are \$US16.65 & -3.30 & 33 & $-10,000$ \\
\hline When costs of single-use EEG-CE are $\$$ US20.35 & -0.40 & 33 & -1212 \\
\hline When costs of single-use EEG-CE are $\$$ US22.20 & -2.25 & 33 & -6818 \\
\hline Two cases of sepsis per 100,000 EEG procedures & 0.78 & 31 & -2516 \\
\hline Four cases of sepsis per 100,000 EEG procedures & 0.1 & 29 & 345 \\
\hline Six cases of sepsis per 100,000 EEG procedures & -0.57 & 27 & 2122 \\
\hline Eight cases of sepsis per 100,000 EEG procedures & -1.25 & 25 & 5000 \\
\hline
\end{tabular}

$E E G$ electroencephalography, $E E G-C E$ electroencephalography cup electrodes, ICER incremental costeffectiveness ratio

${ }^{\text {a }}$ Sepsis cases per 100,000 EEGs

${ }^{\mathrm{b}}$ Cost per avoided sepsis 


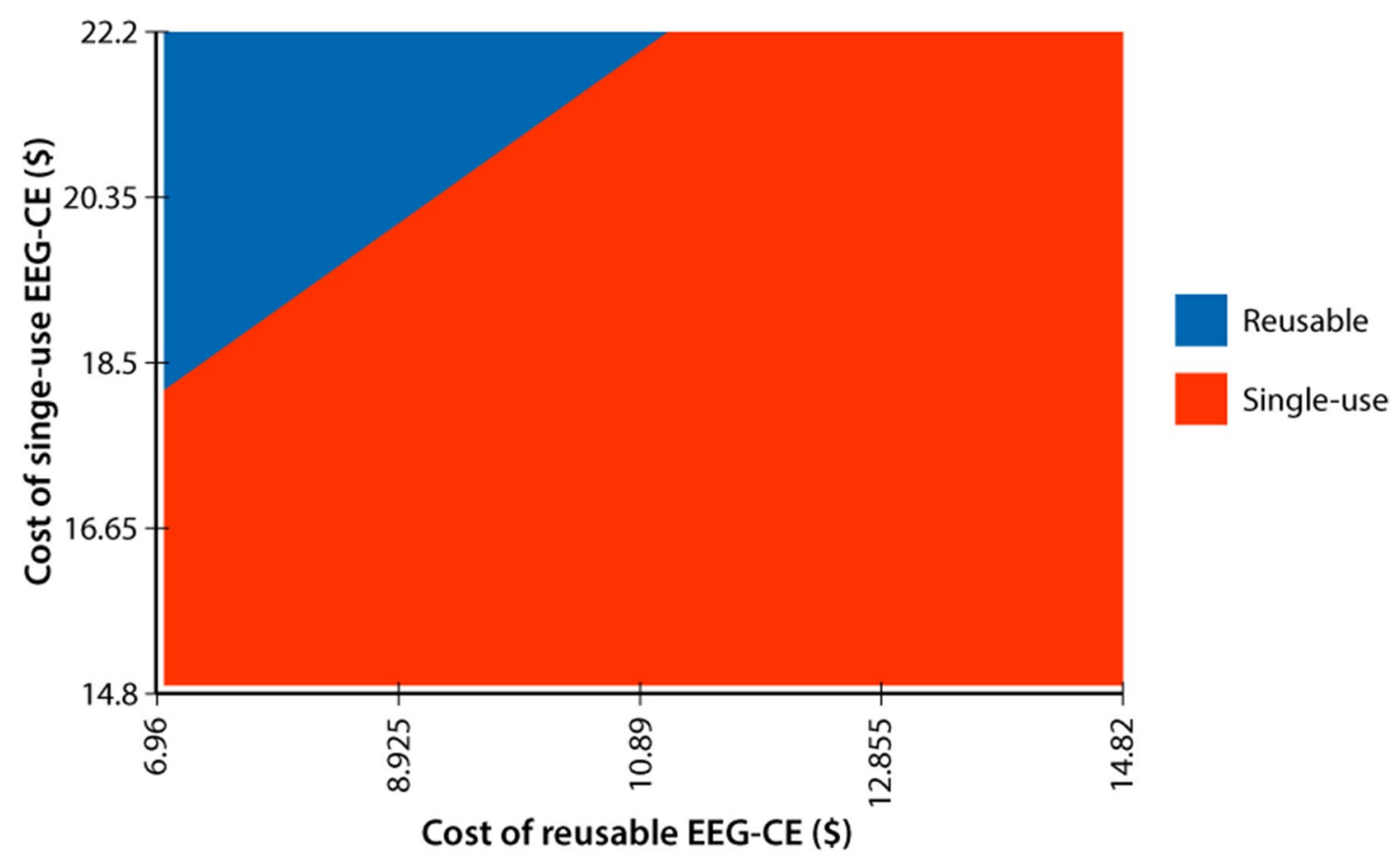

Fig. 2 Two-way sensitivity analysis. The red area indicates savings from using single-use electroencephalography cup electrodes (EEG-CE), and the blue area indicates savings from using reusable EEG-CE

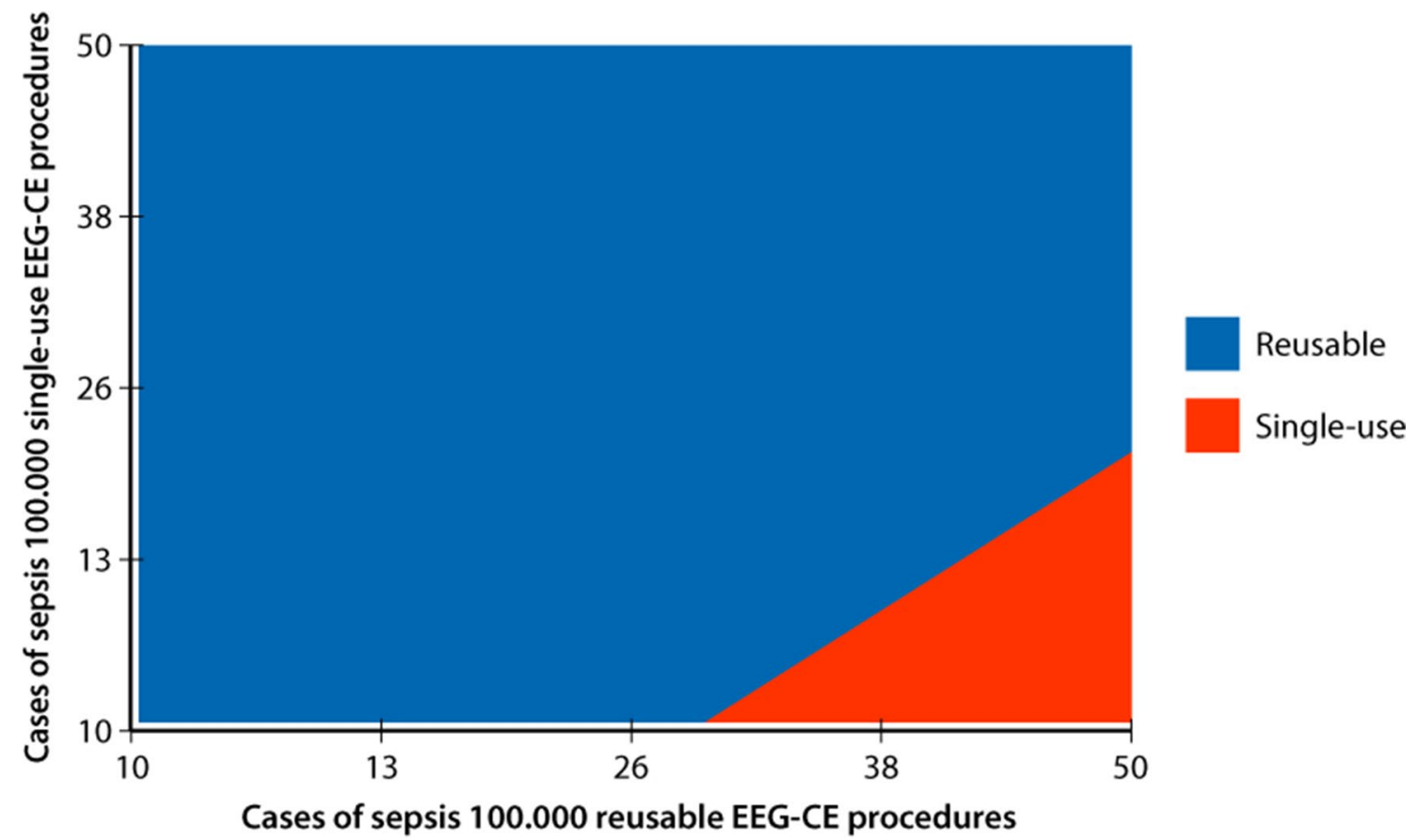

Fig. 3 Two-way sensitivity analysis. The red area indicates savings from using single-use electroencephalography cup electrodes (EEG-CE) and the blue area indicates savings from using reusable EEG-CE 


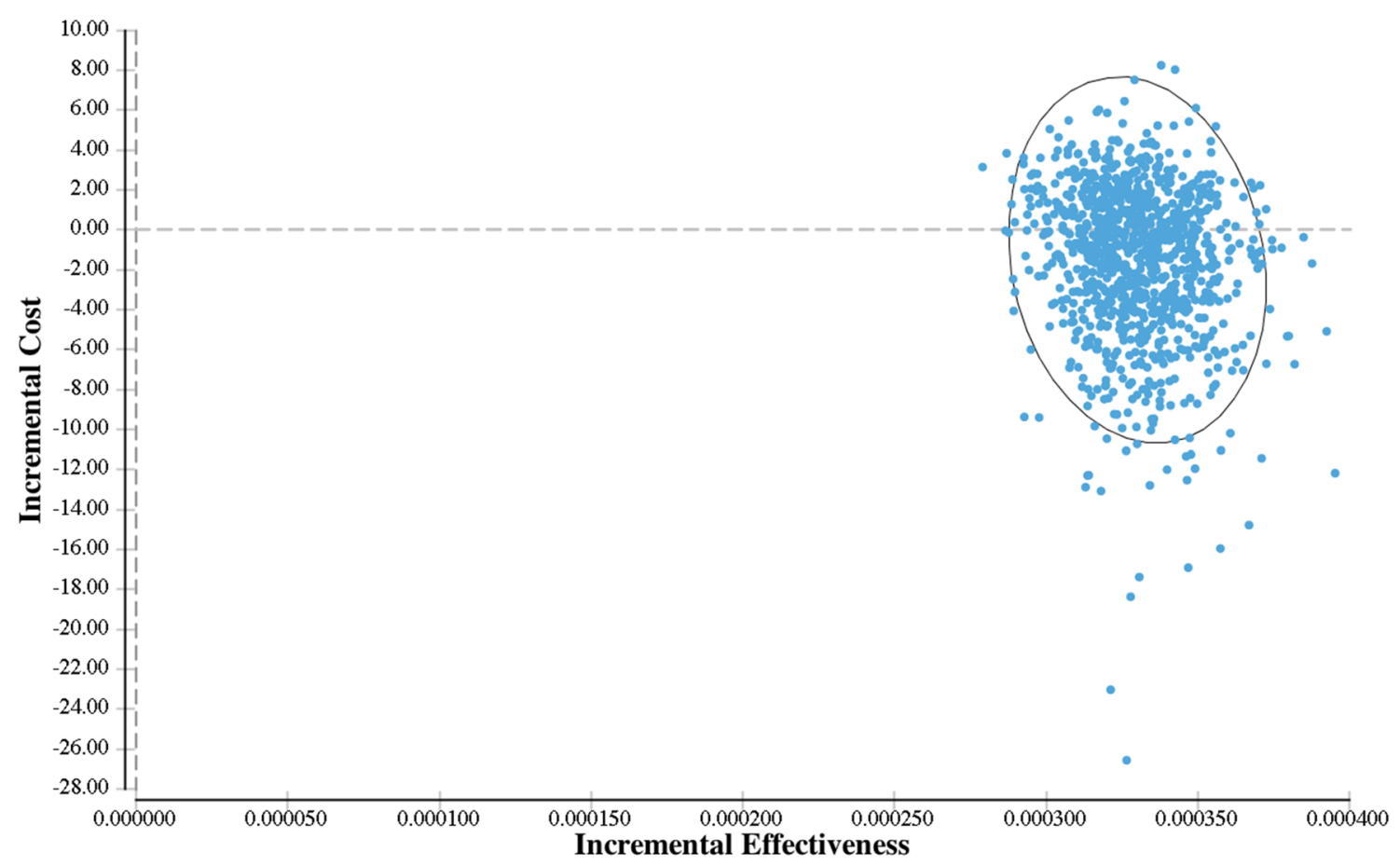

Fig. 4 Scatterplot from the probabilistic sensitivity analysis. $62 \%$ of the trials are located in the south-eastern quadrant

of the calculated ICERs were situated in the south-eastern quadrant and $38 \%$ were in the north-eastern quadrant. Figure 4 is a scatter plot of the PSA result, with an ellipse indicating the $95 \%$ confidence interval.

\section{Discussion}

This study is the first to identify the incidence of sepsis after EEG procedures. There were 33 cases of sepsis per 100,000 EEG procedures, whereas the incidence of sepsis after the non-EEG procedure was assumed to be 0 . Furthermore, this is the first cost-effectiveness analysis to compare reusable and single-use EEG-CE. The base-case result was that single-use EEG-CE were more effective at avoiding sepsis and less costly than reusable EEG-CE, although the PSA showed the result was not robust: $38 \%$ of the ICERs indicated that single-use EEG-CE were more effective in avoiding sepsis but more expensive. Thus, which technology to choose on the basis of cost is unclear; nevertheless, the single-use alternative may significantly reduce the risk of cross-contamination.

This study had some limitations. First, Truven Health Analytics MarketScan ${ }^{\circledR}$ Commercial Claims and Encounters and Medicare Supplemental Databases do not allow device segmentation. Thus, it was assumed that all EEG procedures were conducted with reusable EEG-CE. This is because the vast majority of EEG-CE used in EEG procedures are reusable [17]. The risk of sepsis was assumed to be zero with single-use EEG-CE because they are used right from a sealed pack [17]. Figure 3 illustrates that assuming single-use EEG-CE are associated with a risk of sepsis affects the results. Consequently, the incidence of sepsis is associated with uncertainty, and more research on the correlation between sepsis and EEG procedures is needed.

Second, reprocessing costs of reusable EEG-CE were based on interviews. The reprocessing time was based on estimates and not actual time measurements, and assumptions were made about the amounts and costs of detergent and disinfectants. However, as the reprocessing is not very complex, we assumed the estimates were reliable.

Third, we only considered the risk of sepsis in this study, not the risk of minor infections and skin infections. As sepsis is a progression of an infection, it must be assumed that the risk of other infections is higher than the risk of sepsis. Although the costs related to these infections are significantly lower than those associated with sepsis, patient discomfort must also be considered when choosing a technology.

Additionally, the retrospective observational study assessing the risk of sepsis only included outpatients. However, patients with chronic medical conditions and weakened immune systems are at greater risk of sepsis [12], so it must be assumed that inpatients have a higher risk of sepsis. Hence, this should be considered when using EEG-CE on inpatients. Another limitation is that sepsis, particularly 
severe sepsis, is associated with high mortality rates. A 2014 meta-analysis reported average mortality rates of $29 \%$ among patients with severe sepsis in the USA between 2006 and 2009 [18]. Mortality rates and disutilities were not included in this cost-effectiveness analysis.

Environmental factors were not included in this study. The increased waste disposal and handling associated with disposable single-use EEG-CE may represent an additional cost; in addition, the use of materials is greater, which is not environmentally responsible. This raises ethical considerations: some would think the healthcare system should seek to reduce the consumption of scarce resources and not burden the environment more than necessary; others would argue the safety of the patient should always come first, regardless of the cost.

This study was based on hospitals that use HLD as the reprocessing method. This method is less time consuming and thereby less costly than sterilization. HLD eliminates all pathogenic microorganisms on an instrument except for a small number of bacterial spores, whereas sterilization eliminates all microorganisms, including bacterial spores. Thus, sterilization is a more effective reprocessing method than HLD [9, 10]. In addition, although guidelines as to how HLD should be conducted do exist, how thoroughly the HLD is executed may vary between clinics and people. This contrasts with sterilization, which involves a machine. Thus, it must be assumed that cross-contamination occurs more frequently with HLD than with sterilization. Consequently, costs related to the risk of cross-contamination may be higher when EEG electrodes undergo HLD rather than sterilization. On the other hand, costs of sterilization are assumed to be significantly higher than of HLD.

\section{Conclusion}

The costs of reusable EEG-CE were based on data from four hospitals and are associated with some uncertainty. Uncertainty would be reduced if data no costs and reprocessing time were collected from more sites. Additionally, further research is needed to determine the number of times reusable EEG-CE can be reused, as this is decisive for the acquisition cost.

Acknowledgments The authors thank Nancy Albert and Ellen Slifcak for their contribution to data collection.

Data Availability Statement Cost data from the four US hospital centers are available in the Electronic Supplementary Material.

Author contributions AS contributed to data collection and writing of the manuscript. AM and WVP contributed methodology advice and manuscript drafting and revision. All authors approved the manuscript.

\section{Compliance with Ethical Standards}

Funding This study was funded by Ambu A/S

Conflicts of Interest AS and AM are employed by Ambu A/S. Johns Hopkins Bloomberg School of Public Health received an unrestricted grant from Ambu A/S related to Dr. WVP.

Open Access This article is distributed under the terms of the Creative Commons Attribution-NonCommercial 4.0 International License (http://creativecommons.org/licenses/by-nc/4.0/), which permits any noncommercial use, distribution, and reproduction in any medium, provided you give appropriate credit to the original author(s) and the source, provide a link to the Creative Commons license, and indicate if changes were made.

\section{References}

1. World Health Organization. Report on the Burden of Endemic Health Care-Associated Infection Worldwide. WHO Libr Cat Data; 2011.

2. Burke JP. Infection control - a problem for patient safety. N Engl J Med. 2003;348:651-6.

3. Siegel JD, Rhinehart E, Jackson M, Chiarello L. The Healthcare Infection Control Practices Advisory Committee. 2007 Guideline for Isolation Precautions: Preventing Transmission of Infectious Agents in Healthcare Settings. Centers for Disease Control and Prevention; 2007.

4. Mazzaro N. Ambu disposable cup electrode. 2010. https://www. ambu.com/Files/Images/ambu/clinical-studies/Neuroline_EEG_ Cup/IE-WP-Disposable-cup-electrode-496730001-1110.pdf. Accessed 6 Dec 2017.

5. Hall JE, Guyton AC. Medical physiology. 12th edn. Saunders; 2010. p. 723.

6. Teplan M. Fundamentals of EEG measurement. Meas Sci Rev. 2002;2:1-11.

7. Ferree TC, Luu PL, Russel GS, Tucker DM. Scalp electrode impedance, infection risk, and EEG data quality. J Chem Inf Model. 2013;53:1689-99.

8. US Food and Drug Administration. Medical Devices. What are reusable medical devices? Silver Spring, MD: US FDA; 2015. http://www.fda.gov/MedicalDevices/ProductsandMedicalProcedu res/ReprocessingofReusableMedicalDevices/ucm454619.htm. Accessed 6 Dec 2017.

9. Rutala WA, Weber DJ, The Healthcare Infection Control Practices Advisory Committee (HICPAC). Guideline for Disinfection and Sterilization in Healthcare Facilities, 2008. Centers for Disease Control and Prevention; 2008. https://www.cdc.gov/infectionc ontrol/pdf/guidelines/disinfection-guidelines.pdf. Accessed 6 Dec 2017.

10. Scott NK. Infection prevention: 2013 review and update for neurodiagnostic technologists. Neurodiagn J. 2013;53:271-88.

11. Albert N, Bena J, Runner J, Morrision J, Ciudad, Rice K, Keleekai N, Slifack. Contamination of reusable EEG electrodes, a multicenter Study. 2018 poster presentation at APIC https://www.event scribe.com/2018/APIC/searchbybucket.asp?h=Oral\%20Abstract $\mathrm{s} \& \mathrm{BCFO}=\mathrm{O} \& \mathrm{f}=$ SessionIDNumber. Session 2103.

12. Arefian H, Heublein S, Martin F, Younis MZ, Moerer O, Fischer D, et al. Hospital-related cost of sepsis: a systematic review. J Infect. 2017;74:107-17.

13. Centers for Disease Control and Prevention. Sepsis. 2017. https:// www.cdc.gov/sepsis/basic/index.html. Accessed 6 Dec 2017. 
14. Trading Economics. Consumer Price Index 2017. http://www. tradingeconomics.com/united-states/consumer-price-index-cpi. Accessed 1 Nov 2017.

15. IBM Watson Health www.truvenhealth.com/. Accessed $11 \mathrm{Apr}$ 2018.

16. Ambu A/S www.ambu.com. Accessed 13 Apr 2018.
17. Finnegan C. The case of disposable EEG electrodes. J Electrophysiol Technol. 2007;33:115-9.

18. Stevenson E, et al. Two decades of mortality trends among patients with severe sepsis: a comparative meta-analysis. Crit Care Med. 2014;42(3):625-31. 